\title{
Non-invasive focused ultrasound for abdominal circumference reduction: does it really work?
}

\author{
Eric Barel ${ }^{1,2}$, Abraham Amir ${ }^{1}$, Asaf Olshinka', Dean Ad-El ${ }^{1}$ \\ 'Department of Plastic and Reconstructive Surgery, Rabin Medical Center, Beilinson Hospital (affiliated with Sackler Faculty of Medicine, Tel Aviv \\ University), Petach Tikva 49100, Israel. \\ ${ }^{2}$ Post-Barriatric Plastic Surgery Clinic, Rabin Medical Center, Beilinson Hospital (affiliated with Sackler Faculty of Medicine, Tel Aviv University), Petach \\ Tikva 49100, Israel.
}

Correspondence to: Dr. Eric Barel, Department of Plastic and Reconstructive Surgery, Rabin Medical Center, Beilinson Hospital (affiliated with Sackler Faculty of Medicine, Tel Aviv University), Petach Tikva 49100, Israel. E-mail: eric@drbarel.com

How to cite this article: Barel E, Amir A, Olshinka A, Ad-El D. Non-invasive focused ultrasound for abdominal circumference reduction: does it really work? Plast Aesthet Res 2016;3:368-74.

\section{Article history: \\ Received: 29-07-2015 \\ Accepted: 27-10-2016 \\ Published: 14-12-2016}

\section{Key words:}

Body contouring,

ultrasound,

fat cell disruption,

liposuction,

non-invasive

\section{ABSTRACT}

\begin{abstract}
Aim: Non-invasive body contouring is a promising modality. However, due to a lack of good evidence-based data, the mechanism by which contouring occurs remains unclear. The purpose of this study was to evaluate the effect of treatment with the Contour I ${ }^{\mathrm{TM}}$ ultrasound system (Ultrashape $^{\circledR}$, Syneron ${ }^{\circledR}$, Israel) on abdominal circumference and to compare 2 power levels for efficacy and safety. Methods: A prospective, self-controlled double-blind design was used. Thirty-six women, aged 30-45 years, were randomized to receive treatment with the Contour I at high or low acoustic outputs in 3 successive sessions, 1 month apart. Safety was evaluated by adverse events, local skin reaction, and pain. Efficacy was evaluated by the change in abdominal circumference relative to baseline and to the untreated thigh area (internal control). Patients were followed for 28 days after the last treatment session. Results: At 1 month after the first session, the mean reductions in abdominal circumference measured $1.65 \mathrm{~cm}(P<0.001)$ and $0.87 \mathrm{~cm}$ $(P<0.019)$ in the high and low-power groups, respectively. At 1 month after the last session, the cumulative reductions in circumference were $2.56 \mathrm{~cm}(P<0.001)$ and $1.49 \mathrm{~cm}(P<0.012)$, respectively. There was no change in the internal-control circumference throughout treatment. There were no treatment-induced severe adverse events. Conclusion: Multiple successive treatments of the abdominal area with the Contour I lead to a significant progressive reduction in circumference. The magnitude of the reduction is directly correlated to the acoustic power output which suggests that the technology itself is the main cause for the contouring effect.
\end{abstract}

\section{INTRODUCTION}

Body contouring is one of the most popular procedures in aesthetic medicine. There is a growing demand for non-invasive alternatives to traditional liposuction given the risk of complications ${ }^{[1-5]}$ and prolonged downtime. ${ }^{[6]}$ Multiple modalities have been developed including the use of laser and radio-frequency assisted liposuction,

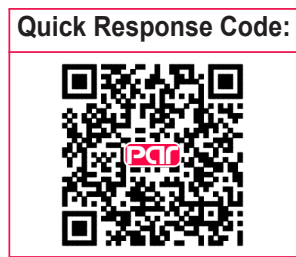


but these modalities are minimally invasive, painful, and with unproven efficacy. There is still a demand for a true noninvasive modality. Ultrasound has served as a therapeutic tool for more than 50 years. ${ }^{[7,8]}$ Highpower, high-intensity ultrasound is used for ablation and lithotripsy, and low-power ultrasound is used for sonophoresis, sonoporation, gene therapy, and bone healing.$^{[9]}$ The underlying mechanism of action is thermal or mechanical.

The Contour I ${ }^{\mathrm{TM}}$ (Ultrashape $^{\circledR}$, Syneron ${ }^{\circledR}$, Yokneam, Israel) is an FDA-approved, non-invasive, focused ultrasound device designed for body contouring. It selectively and mechanically disrupts adipocytes in the subcutaneous fat layer by a process of cavitation without damaging neighboring blood vessels, nerves, or muscles. It is based on a unique design of energy spreading that is focused $1.5 \mathrm{~cm}$ beneath the skin for selective targeting of the fat layer without affecting the dermis or deep structures. Its safety and efficacy have been evaluated in several pre-clinical and clinical studies. ${ }^{[10-14]}$ Treatment requires no anesthesia and may be performed in a non-surgical setting.

Although its efficacy has been shown in numerous studies, as with other modalities, the main factors contributing to the body contouring effect are not clear and can be attributed to other aspects such as skin tightening, inflammatory response and lifestyle change during the treatment period.

The aim of the present study was to evaluate the cumulative effect of successive treatments with the Contour $\mathrm{I}^{\mathrm{TM}}$ device system on abdominal circumference and to compare two acoustic power levels for efficacy and safety.

\section{METHODS}

\section{Setting}

A prospective self-controlled, double-blinded trial was performed. The study was approved by the Helsinki Committee of Rabin Medical Center and Sackler Faculty of Medicine, Tel Aviv University, and was conducted in accordance with the Declaration of Helsinki and the International Conference of Harmonization/Good Clinical Practice (ICH-GCP) guidelines. Informed consent for participation in the study was obtained from participants.

\section{Patients}

The study cohort consisted of healthy Caucasian women who sought to reduce their abdominal circumference for aesthetic reasons. Inclusion criteria were age $18-50$ years, body mass index $\leq 26 \mathrm{~kg} / \mathrm{m}^{2}$ and abdominal subcutaneous adipose tissue thickness $\geq 1.5 \mathrm{~cm}$, assessed by manual pinch test. The $1.5 \mathrm{~cm}$ criteria was instituted for procedural efficacy and safety according to the Contour $\mathrm{I}^{\mathrm{TM}}$ mechanical specifications (i.e. the focus of the ultrasonic energy). Exclusion criteria included the presence of any systemic chronic disease, blood coagulopathy or excessive bleeding, history of skin disease in the treatment area, known tendency to form keloids, poor wound healing, skin lesions in the treatment area other than simple nevi, depressed scar in the treatment area, abdominal wall diastasis or hernia on physical examination, and abnormalities in kidney or liver function, lipid profile, or blood count in the last 3 months. Women who were pregnant or lactating at baseline or during the study period and women who had given birth within the last 12 months were also excluded.

\section{Procedure}

Treatments were administered to the anterior abdominal area with the Contour I ${ }^{\mathrm{TM}}$ system, aided by computerized video tracking [Figure 1]. The targeted area was the lower abdomen, which was marked prior to the procedure. The procedure was performed with the patient in a flat supine position. The treated area is captured by the video system and is automatically divided into points of treatment that are followed by the trained operator of the device. ${ }^{[10]}$ Subjects were randomized to receive high acoustic power output $\left(\right.$ Isppa $\left.=440 \mathrm{~W} / \mathrm{cm}^{2}\right)$ or low acoustic power (Isppa $=370 \mathrm{~W} / \mathrm{cm}^{2}$ ). These parameters were chosen as both extremes of the device's power output. The focal depth for the selected output was $1.5 \mathrm{~cm}$; the exclusion criteria of abdominal subcutaneous adipose tissue thickness $\geq 1.5 \mathrm{~cm}$ was set for this reason. All subjects underwent 3 treatment sessions, 1 month apart, performed by a trained physician. All patients were divided arbitrarily between the high and the low acoustic power groups. Subjects and physician were blinded to the group allocations, as the device was preset before treatment (by a technician), according to the allocation of the subject, and the settings were

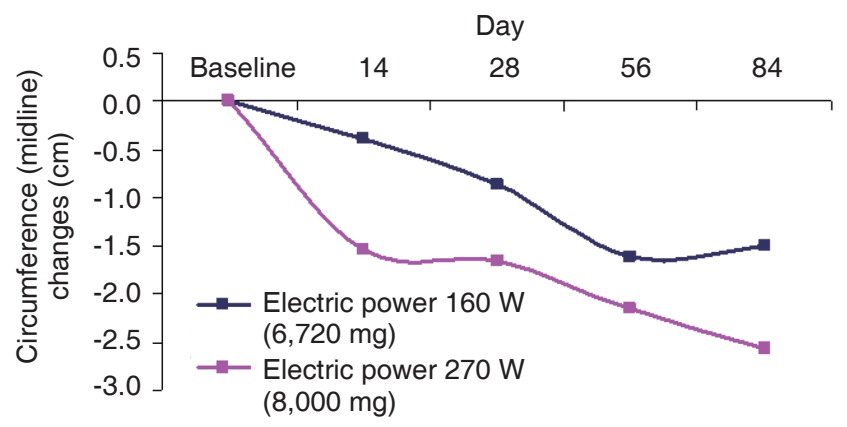

Figure 1: Circumference changes from baseline by day and study group in treatment area 
not shown on the device's control panel.

\section{Abdominal circumference measurement}

At baseline (prior to treatment, day 1), each subject's abdominal circumference was measured independently by 3 trained operators who were blinded to the group allocation. All used the same standardized calibrated tape according to the same standardized and validated technique, ensuring that subject positioning, posture, and breathing were consistent for all measurements. All measurements were performed in the same anatomical spots, while standing in a standardized position. On days 14 (2 weeks after the first treatment), 28 (4 weeks after the first treatment), 56 ( 4 weeks after the second treatment), and 84 (4 weeks after the third treatment), measurements were repeated in the same manner, at the same height (per individual), in duplicate. The circumference of the untreated thigh area was also measured at all visits to serve as a control measurement.

\section{Adverse events and patient satisfaction}

At each follow-up visit, subjects were examined for adverse events related to the treatment, and local skin reaction. Adverse events were recorded as Medical Dictionary for Regulatory Activities (MedDRA) codes. In addition, the subjects were asked to complete a satisfaction questionnaire, as follows:

(1) Has there been a visible change in your body contour since the beginning of the study? (yes, favorable change/ no change/yes, unfavorable change)

(2) Have other people commented on a change in your bodily appearance? (yes, favorably/no comments/yes, unfavorably)

(3) Would you recommend this procedure to your friends? (yes/no)

(4) Is the Contour I ${ }^{\mathrm{TM}}$ system preferable to liposuction? (yes/no)

(5) How would you grade pain from the treatment on a scale of 1-10?

\section{Statistical analysis}

Data were analyzed with SAS software, version 9.1 (SAS Institute, Cary, NC). Paired $t$-test and nonparametric signed-rank test were used to analyze differences in circumference within each study group by time. Student $t$-test and Wilcoxon rank rest were used to analyze differences in the reduction in circumference at each time point between the study groups. MantelHaenszel chi-square test or linear regression was used to examine $P$ values or trends during the study and follow-up periods. All tests applied were two-tailed. $P$ values of $5 \%$ or less were considered statistically significant.
Table 1: Subject demographic characteristics

\begin{tabular}{|c|c|c|c|}
\hline Char & $\begin{array}{c}\text { High power } \\
\left(\text { Isppa } 440 \mathrm{~W} / \mathrm{cm}^{2}\right)\end{array}$ & $\begin{array}{c}\text { Low power } \\
\left(\text { Isppa } 370 \mathrm{~W} / \mathrm{cm}^{2}\right)\end{array}$ & $\begin{array}{c}P \\
\text { value }\end{array}$ \\
\hline 50 & 19 & 17 & \\
\hline & $02 \pm$ & 34 & 0.0407 \\
\hline & & & 0.2804 \\
\hline $\mathrm{BM}$ & ? & 23 & 0.2786 \\
\hline & $164.26 \pm 1.43$ & $164.12 \pm 1.22$ & 0.9396 \\
\hline Fat thickness, $\mathrm{mm}$ & $25.87 \pm 1.70$ & $27.15 \pm 1.98$ & 0.6247 \\
\hline
\end{tabular}

Data shown as mean \pm SE. BMI: body mass index

\section{RESULTS}

\section{Subject disposition and baseline demographic characteristics}

All 36 subjects completed the study protocol with 19 in the high-power group and 17 in the low-power group. There were no between-group differences in mean weight, height, body mass index, or abdominal fat thickness [Table 1]. Ages ranged from 30 to 45 years. Mean age was significantly lower in the low-power than the high-power group (34 vs. 38 years, $P=0.04$ ), but this 4-year difference had no clinical relevance with respect to abdominal circumference reduction.

\section{Objective efficacy endpoints}

Both study groups showed a statistically significant reduction in circumference of the treated area compared to baseline at all time points, with the exception of the low-power group on day $14(P=0.113)$, and a cumulative reduction over time [Table 2 and Figure 1]. On day 28 (4 weeks after the first treatment), the mean reduction in abdominal circumference was $1.65 \mathrm{~cm}$ in the high-power group $(P<0.001)$ and $0.87 \mathrm{~cm}$ in the low-power group $(P=0.019)$. On day 56 (1 month after the third treatment session), the mean reductions in the respective groups were $2.14 \mathrm{~cm}(P=0.002)$ and $1.62 \mathrm{~cm}(P<0.001)$, and on day $84,2.56 \mathrm{~cm}(P<0.001)$ and $1.49 \mathrm{~cm}(P=0.012)$ [Table 2 and Figure 1]. The subjects' weight remained generally constant during the entire period of the study; the overall change in weight did not exceed $1.6 \%$ of baseline, with no between-group difference [Table 2].

The high-power group showed a greater and a more consistent reduction in abdominal circumference than the low-power group, but the difference did not reach statistical significance.

Analysis of the control (thigh) areas yielded an increase in circumference in both groups at all study time points [Table 3 and Figure 2], with the exception of the lowpower group on day 14 (no change). There was no statistically significant difference in mean internalcontrol circumference between the groups (data not shown). However, a statistically significant difference 
Table 2: Circumference changes from baseline by day and study group in treatment area

\begin{tabular}{|c|c|c|c|c|c|c|c|c|c|}
\hline \multirow{2}{*}{$\begin{array}{l}\text { Change from } \\
\text { baseline }\end{array}$} & \multicolumn{4}{|c|}{ High power (Isppa $440 \mathrm{~W} / \mathrm{cm}^{2}$ ) } & \multicolumn{4}{|c|}{ Low power (Isppa $370 \mathrm{~W} / \mathrm{cm}^{2}$ ) } & \multirow{2}{*}{$\begin{array}{c}\begin{array}{c}\text { Weight differences } \\
\text { between groups }\end{array} \\
\boldsymbol{P} \text { value }\end{array}$} \\
\hline & Number & Mean $(\mathrm{cm})$ & SE & $P$ value & Number & Mean $(\mathrm{cm})$ & SE & $P$ value & \\
\hline Day 14 & 19 & -1.54 & 0.57 & 0.016 & 17 & -0.39 & 0.24 & 0.113 & 0.8741 \\
\hline Day 28 & 19 & -1.65 & 0.42 & $<0.001$ & 17 & -0.87 & 0.33 & 0.019 & 0.9317 \\
\hline Day 56 & 19 & -2.14 & 0.60 & 0.002 & 17 & -1.62 & 0.36 & $<0.001$ & 0.9981 \\
\hline Day 84 & 19 & -2.56 & 0.63 & $<0.001$ & 17 & -1.49 & 0.52 & 0.012 & 0.9896 \\
\hline
\end{tabular}

Table 3: Circumference changes from baseline by day and study group in control area (untreated thighs)

\begin{tabular}{|c|c|c|c|c|c|c|c|c|}
\hline \multirow{2}{*}{ Change from baseline } & \multicolumn{4}{|c|}{ High power (Isppa $440 \mathrm{~W} / \mathrm{cm}^{2}$ ) } & \multicolumn{4}{|c|}{ Low power (Isppa $370 \mathrm{~W} / \mathrm{cm}^{2}$ ) } \\
\hline & Number & Mean $(\mathrm{cm})$ & SE & $P$ value & Number & Mean $(\mathrm{cm})$ & SE & $P$ value \\
\hline Day 14 & 19 & 0.32 & 0.31 & 0.316 & 17 & -0.02 & 0.24 & 0.933 \\
\hline Day 28 & 19 & 0.47 & 0.37 & 0.214 & 17 & 0.17 & 0.24 & 0.484 \\
\hline Day 56 & 19 & 0.60 & 0.26 & 0.033 & 17 & 0.60 & 0.42 & 0.176 \\
\hline Day 84 & 19 & 1.08 & 0.32 & 0.003 & 17 & 1.25 & 0.48 & 0.019 \\
\hline
\end{tabular}

Table 4: Results of subject satisfaction feedback questionnaire

\begin{tabular}{|c|c|c|c|c|c|c|}
\hline \multirow{2}{*}{ Questions in questionnaire } & \multicolumn{2}{|c|}{ Day 28} & \multicolumn{2}{|c|}{ Day 56} & \multicolumn{2}{|c|}{ Day 84} \\
\hline & High power & Low power & High power & Low power & High power & Low power \\
\hline $\begin{array}{l}\text { Subjects reported favorable change in body } \\
\text { contour since beginning of study }\end{array}$ & $27.8 \%$ & $41.2 \%$ & $61.1 \%$ & $64.7 \%$ & $68.4 \%$ & $58.8 \%$ \\
\hline $\begin{array}{l}\text { Subjects reported receiving comments from } \\
\text { other people regarding their appearance }\end{array}$ & $27.8 \%$ & $41.2 \%$ & $72.2 \%$ & $70.6 \%$ & $57.9 \%$ & $70.6 \%$ \\
\hline $\begin{array}{l}\text { Subjects reported to recommend this } \\
\text { procedure to their friends }\end{array}$ & $77.8 \%$ & $76.5 \%$ & $72.2 \%$ & $76.5 \%$ & $68.4 \%$ & $70.6 \%$ \\
\hline $\begin{array}{l}\text { Subjects reported to prefer the Contour ITM } \\
\text { treatments over a short-term body contouring } \\
\text { procedure }\end{array}$ & $66.7 \%$ & $70.6 \%$ & $66.7 \%$ & $64.7 \%$ & $89.5 \%$ & $70.6 \%$ \\
\hline
\end{tabular}

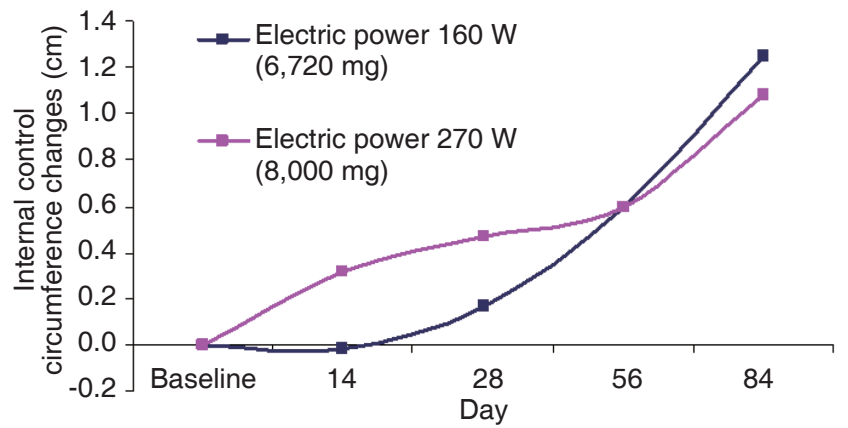

Figure 2: Circumference changes from baseline by day and study group in control area (untreated thighs)

was observed in the relative change between the treated and untreated areas at all time points, with the exception of the low-power group on day 14 .

Representative photographs of abdominal circumference before and after treatment are shown [Figure 3].

\section{Subject satisfaction}

Most subjects felt no pain or only minimal pain during all treatments, 92 of 108 documented encounters $(85.1 \%)$. The high-power group reported a slightly greater pain sensation when compared to the lowpower group: rates of no pain or minimal pain reported by the high-power group were $79.0 \%$ after the first treatment, $84.2 \%$ after the second treatment, and $84.2 \%$ after the third; corresponding values in the low-power group were $94.1 \%, 82.3 \%$, and $88.2 \%$. "Unbearable" pain sensation was uncommon across all treatments and modalities. "Unbearable" pain sensation at all 3 sessions was reported by one subject in the low-power group, at the first session by 2 subjects in the high-power group, and at the second and third sessions by one subject in the high-power group. All reports were obtained after the treatments were done, and no treatment had to be stopped secondary to pain. Nevertheless, all subjects who reported "unbearable" pain completed all treatments and follow-up procedures as scheduled.

Evaluation of the feedback questionnaire revealed that on day 28 , in response to the item on visible change in appearance, a favorable change was reported by $27.8 \%$ of the high-power group and $41.2 \%$ of the lowpower group [Table 4]. On day 56, these rates rose to $61.1 \%$ and $64.7 \%$, respectively. On day 84,1 month following third treatment, $68.4 \%$ of the high-power group reported a positive visible change compared to $58.8 \%$ of the low-power group [Table 4]. At the same time point, $57.9 \%$ of the high-power group reported positive comments from others, and $68.4 \%$ claimed they would recommend the procedure to their friends; 

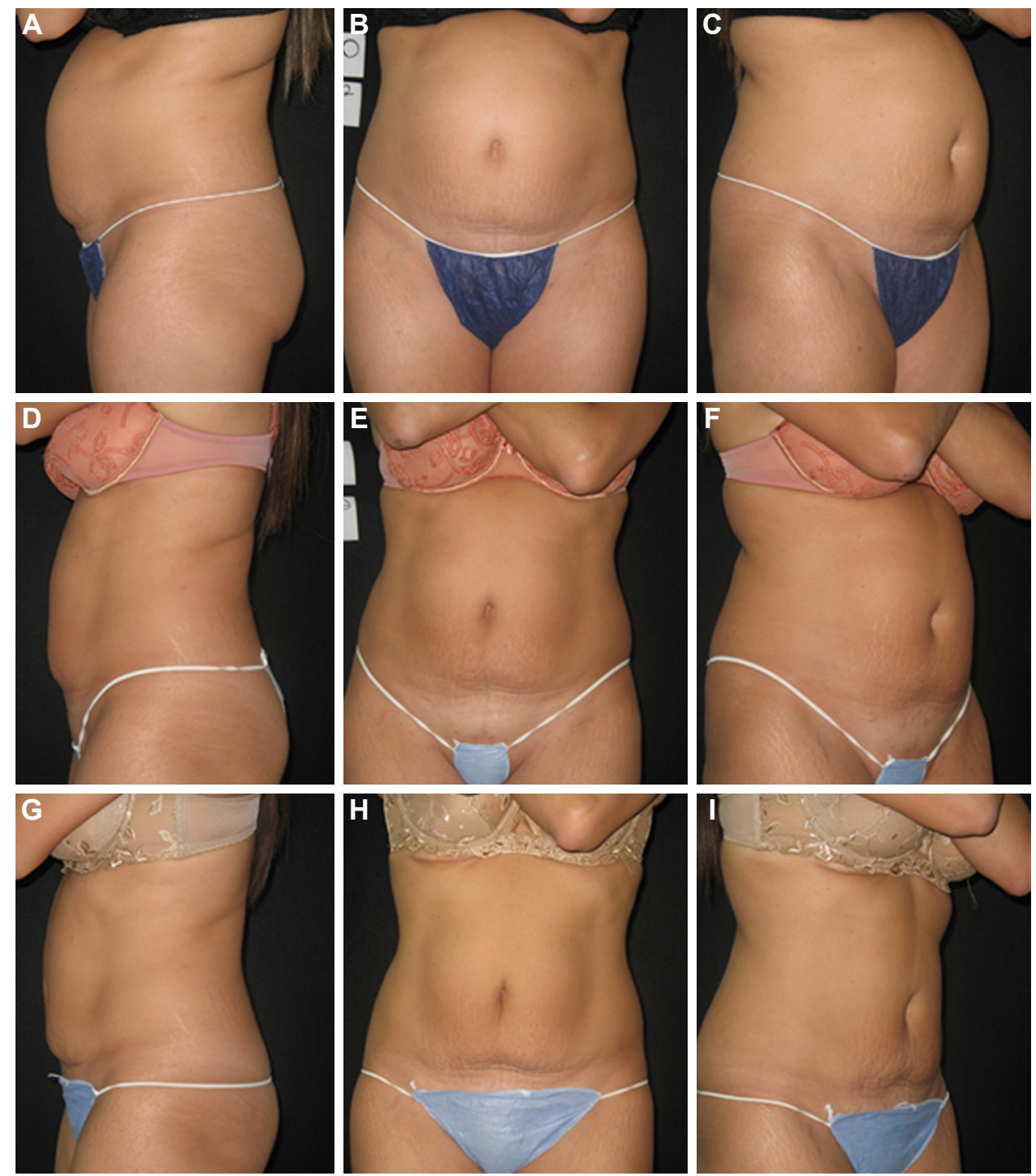

Figure 3: Representative photographs before $(A-C)$ and after (D-I) treatments with the Contour ITM at high power $\left(I s p p a=440 \mathrm{~W} / \mathrm{cm}^{2}\right)$ in a single subject. Abdominal circumference decreased by $3.1 \mathrm{~cm}$ from baseline at 1 month after first treatment (D-F) and an additional $3.3 \mathrm{~cm}$ after 2 additional treatments (G-I) (total reduction, $6.4 \mathrm{~cm}$ ). The reduction from baseline in the untreated control (thigh) area was $0.4 \mathrm{~cm}$ at completion of treatment

the corresponding rates in the low-power group were both $70.6 \%$ [Table 4]. More subjects in the high-power group preferred the Contour $\mathrm{I}^{\mathrm{TM}}$ over liposuction $(89.5 \%$ vs. $70.6 \%)$ [Table 4$]$.

\section{Safety endpoints}

Adverse events included erythema, petechial rash, folliculitis, and hyperesthesia. Six subjects (16.7\%) reported mild to moderate adverse events, 3 in each group. There was no relationship between the occurrence of adverse events and the acoustic power of the ultrasound. The rate of adverse events per treatment sessions was $5.5 \%$. All adverse events resolved spontaneously by the end of the study, with no need for medical intervention. In no case were the adverse events a reason for terminating a treatment.

\section{DISCUSSION}

In the search for the gold standard of non-invasive technology for body contouring, ultrasound energy is a promising tool. The present study evaluated the cumulative effect of successive treatments with the Contour $\mathrm{I}^{\mathrm{TM}}$ ultrasound system on abdominal circumference, with comparison of two acoustic output levels: $440 \mathrm{~W} / \mathrm{cm}^{2}$ (high power) and $370 \mathrm{~W} / \mathrm{cm}^{2}$ (low power). Prior studies have shown that the ultrasonic energy delivered by the Contour I ${ }^{\mathrm{TM}}$ system disrupts cells in the histological level and causes a transient increase in blood lipids.

This study had a few limitations that should be noted. Although statistically significant, the number of participating patients is not very large, and subject 
variability, it terms of skin quality and elasticity, can have an effect. In addition, circumference measurement is a rather crude measurement. None of the patients underwent prior liposuction, and therefore no comparison can be made with invasive body contouring of any kind. Another important factor is that patient's lifestyle effect was not a part of this study; the only control was weight and thigh circumference. No strict protocol for diet and physical activity was taken into consideration. A final item for consideration is that study-related patient satisfaction in aesthetic procedures may not be comparable to real-life data from patients who have actually paid for the procedure.

The findings indicate that both power levels, with 3 treatment sessions, led to a statistically significant reduction in circumference of the treated area relative to baseline. Reductions were noted after each treatment session and overall, after all sessions were completed. The high-power setting was more effective than the low-power setting in reducing the circumference of the treated area after the 1st session and after the 3rd session.

As mentioned, both study groups showed a statistically significant reduction in circumference of the treated area compared to baseline at all time points, with the exception of the low-power group on day $14(P=0.113)$. Our assumption is that this data results from tissue edema.

The decision to use circumference measurement only, and not imaging studies, was based on two reasons. First, subjects normally measure their circumference by wearing trousers or skirts, and the aim of the treatment is to produce erect posture contouring. Secondly, the supine posture in magnetic resonance imaging or ultrasound scan does not correlate exactly with circumference measurement in the erect position since fat distributes differently in the supine position. Later studies done by Ultrashape ${ }^{\circledR}$ have failed to show a significant correlation between these two measurements.

To isolate the net effect of the device on the specific area treated and to eliminate the possibility of bias in the interpretation of the results, the circumference of the untreated thighs from each subject was used as an internal control. The results showed that the reduction in circumference of the treated area relative to the untreated area (true net effect) was statistically significant at all-time points. This finding was supported by the cumulative effect of the multiple treatments on the reduction in circumference of the treated area as well as the correlation between the power output and the magnitude of the effect. An interesting finding was a minimal increase of thigh circumference in some groups. It is unclear if beginning a process of body contouring encourages patients to change their life-style to achieve better overall results, or if it permits them to continue their current habits while relying on technology to correct excessive food intake and a lack of physical activity. The low number of adverse events in our cohort is consistent with the high safety profile of the device reported in extensive pre-clinical ${ }^{[10]}$ and clinical studies. $^{[11-14]}$

Subjective feelings of the patients play a critical role in the outcome of aesthetic procedures, aside from objective evidence of the safety and effectiveness. Therefore, subjects completed a questionnaire evaluating their satisfaction with the results as well as the perception of others. Less than half the subjects in both groups noted a favorable visible change following the first treatment. However, on later follow-up visits, those rates nearly doubled.

In summary, multiple treatments over time with the Contour $\mathrm{I}^{\mathrm{TM}}$ device lead to a cumulative reduction in abdominal circumference. The effect is greater when high acoustic power is used. Patient satisfaction was found to be related to the measured circumference reduction. The findings support the use of the noninvasive Contour I ${ }^{\mathrm{TM}}$ as a safe, painless noninvasive means for body contouring with proven efficacy in circumference reduction, correlated to the power of ultrasonic energy delivered. Due to the limitation of the study, more studies, comparing different modalities in non-invasive body contouring, are needed. Objective non-positional related measurements in form of accurate three-dimensional photography with volume calculation may be of future assistance.

\section{Financial support and sponsorship}

The study was supported by Ultrashape ${ }^{\circledR}$, Syneron $^{\circledR}$. The study sponsors participated in study design and analysis of data.

\section{Conflicts of interest}

There are no conflicts of interest.

\section{Patient consent}

Informed consent was obtained from participants.

\section{Ethics approval}

The study was approved by the Helsinki Committee of Rabin Medical Center and Sackler Faculty of Medicine, Tel Aviv University. 


\section{REFERENCES}

1. Kelley DE. Thermodynamics, liposuction, and metabolism. $N$ Engl J Med 2004;350:2542-4.

2. Kenkel JM, Lipschitz AH, Shepherd G, Armstrong VW, Streit F, Oellerich M, Luby M, Rohrich RJ, Brown SA. Pharmacokinetics and safety of lidocaine and monoethylglycinexylidide in liposuction: a microdialysis study. Plast Reconstr Surg 2004;114:516-24; discussion 525-6.

3. Lipschitz AH, Kenkel JM, Luby M, Sorokin E, Rohrich RJ, Brown SA. Electrolyte and plasma enzyme analyses during large-volume liposuction. Plast Reconstr Surg 2004;114:766-75; discussion 776-7.

4. Rohrich RJ, Leedy JE, Swamy R, Brown SA, Coleman J. Fluid resuscitation in liposuction: a retrospective review of 89 consecutive subjects. Plast Reconstr Surg 2006;117:431-5.

5. Trott SA, Beran SJ, Rohrich RJ, Kenkel JM, Adams WP Jr, Klein KW. Safety considerations and fluid resuscitation in liposuction: an analysis of 53 consecutive subjects. Plast Reconstr Surg 1998;102:2220-9.

6. Co AC, Abad-Casintahan MF, Espinoza-Thaebtharm A. Submental fat reduction by mesotherapy using phosphatidylcholine alone vs phosphatidylcholine and organic silicium: a pilot study. $J$ Cosmet Dermatol 2007;6:250-7.
7. Fry WJ, Mosberg WH Jr, Barnard JW, Fry FJ. Production of focal destructive lesions in the central nervous system with ultrasound. $J$ Neurosurg 1954;11:471-8.

8. Illouz YG. Body contouring by lipolysis: a 5-year experience with over 3000 cases. Plast Reconstr Surg 1983;72:591-7.

9. Illouz YG. Illouz's technique of body contouring by lipolysis. Clin Plast Surg 1984;11:409-17.

10. Brown SA, Greenbaum L, Shtukmaster S, Zadok Y, Ben-Ezra S, Kushkuley L. Characterization of nonthermal focused ultrasound for noninvasive selective fat cell disruption (lysis): technical and preclinical assessment. Plast Reconstr Surg 2009;124:92-101.

11. Ascher B. Safety and efficacy of UltraShape Contour I treatments to improve the appearance of body contours: multiple treatments in shorter intervals. Aesthet Surg J 2010;30:217-24.

12. Coleman KM, Coleman WP 3rd, Benchetrit A. Non-invasive, external ultrasonic lipolysis. Semin Cutan Med Surg 2009;28:263-7.

13. Moreno-Moraga J, Valero-Altés T, Riquelme AM, Isarria-Marcosy MI, de la Torre JR. Body contouring by non-invasive transdermal focused ultrasound. Lasers Surg Med 2007;39:315-23.

14. Teitelbaum SA, Burns JL, Kubota J, Matsuda H, Otto MJ, Shirakabe Y, Suzuki Y, Brown SA. Non invasive body contouring by focused ultrasound: safety and efficacy of the Contour I device in a multicenter, controlled, clinical study. Plast Reconstr Surg 2007;120:779-89. 